\title{
ELEMENTOS PARA UNA PROPUESTA \\ DE POLÍTICA PÚBLICA EN GESTIÓN \\ COMUNITARIA DEL AGUA 1
}

\author{
Raúl Fernando Núñez Marín ${ }^{(a)}$ \\ Felipe Valencia Serrano(b)
}

KEY ASPECTS IN A PUBLIC POLICY PROPOSAL FOR

COMMUNITY WATER MANAGEMENT

ELEMENTOS PARA UMA PROPOSTA DE POLÍTICA

PÚBLICA EM GESTÃO COMUNITÁRIA DA ÁGUA

Fecha de recepción: 28 de febrero del 2020

Fecha de aprobación: 24 de junio del 2020

Disponible en línea: 30 de junio del 2020

Sugerencia de citación:

Núñez Marín, R. F. y Valencia Serrano, F. (2020). Elementos para una propuesta de política pública en gestión comunitaria del agua. Razón Crítica, 9, 159-186. doi: 10.21789/25007807.1622

$1 \quad$ El presente es un artículo de revisión que se estructura a partir del trabajo de investigación titulado "Desarrollo de una política pública para la garantía del derecho humano al agua de las comunidades campesinas", investigación adelantada en el marco del semillero Antonio Cançado Trindade de la Pontificia Universidad Javeriana de Cali, en el que se analiza y discute la necesidad de una política pública diferencial en materia de gestión comunitaria del agua.

(a) Raúl Fernando Núñez Marín

Magister en Derecho de la Universidad Sergio Arboleda

Procurador Regional del Valle del Cauca, Colombia rfnunezm@gmail.com

https://orcid.org/0000-0003-1987-4114

(b) Felipe Valencia Serrano

Abogado de la Pontificia Universidad Javeriana

Socio en KBSV Abogados S.A.S, Colombia

felipevalencia30@gmail.com

https://orcid.org/0000-0002-9275-2239 


\section{R E S U M E N}

El presente artículo expone la necesidad de una política pública en Colombia con enfoque de derechos humanos, que promueva y fortalezca la gestión comunitaria del agua en las zonas rurales del país, dado que esta se ha visto amenazada por el olvido gubernamental y el modelo neoliberal de libertad económica regulada desarrollado en la Ley 142 de 1994. Con este propósito se empleó una metodología hermenéutica y analítica, para la proposición de categorías que permitieran la comprensión histórica y jurídica de las formas de gestión pública, privada y comunitaria del recurso hídrico. Así, los resultados que se obtuvieron permitieron presentar un panorama sobre la gestión comunitaria del agua y la carga regulatoria que deben asumir los acueductos comunitarios en Colombia. Tema que demanda una respuesta estatal estructurada y centrada en el reconocimiento del derecho fundamental al agua, el cumplimiento de las obligaciones que se derivan de instrumentos jurídicos nacionales e internacionales de derechos humanos y la afirmación de las comunidades campesinas y rurales como sujetos colectivos de derechos que tienen una relación especial con el territorio y el recurso hídrico, por lo que deben ser consultados en el diseño e implementación de esta política pública.

PALABRAS CLAVE: Política pública, derecho al agua, gestión comunitaria del agua. 


\section{A B S T R A C T}

This work exposes the need for a human rights-oriented public policy that promotes and strengthens community water management in rural areas of Colombia; an issue that has been neglected by the government and the neoliberal model of regulated economic freedom developed after Law 142 of 1994. For this purpose, a hermeneutic and analytical methodology was implemented in order to propose a series of categories that enable the historical and legal understanding of public, private and community management approaches to water resources. Results provide an overview of community water management and of the regulatory burden that community aqueducts must assume. This situation requires a structured state response focused on the recognition of the fundamental right to water access, the compliance of obligations stated in national and international legal instruments for ensuring human rights, and the assertion of rural and peasant communities as collective subjects of rights with a special bond with their territories and water resources, so they must be previously consulted for the design and implementation of such policy.

KEYWORDS: Public policy, human right to water and sanitation, community water management.

\section{R E S U M O}

Este artigo expõe a necessidade de uma política pública na Colômbia com abordagem em Direitos Humanos que promova e fortaleça a gestão comunitária da água nas áreas rurais do país, tendo em vista que esta tem sido ameaçada pelo esquecimento governamental e pelo modelo neoliberal de liberdade econômica regularizada desenvolvido na Lei 142 de 1994. Com esse objetivo, foi utilizada uma metodologia hermenêutica e analítica para propor categorias que permitiram a compreensão histórica e jurídica das formas de gestão pública, privada e comunitária do recurso hídrico. Assim, os resultados apresentam um panorama sobre a gestão comunitária da água e os tributos que as empresas de saneamento básico comunitárias na Colômbia devem assumir. Tema que requer uma resposta do Estado estruturada e focada no reconhecimento do direito fundamental à água, no cumprimento das obrigações que são derivadas de instrumentos jurídicos nacionais e internacionais de direitos humanos e na afirmação das comunidades camponesas e rurais como sujeitos coletivos de direitos que têm uma relação especial com o território e com o recurso hídrico, por isso devem ser consultados no desenho e implementação dessa política pública.

PALAVRAS-CHAVE: Política pública, direito à água, gestão comunitária da água. 


\section{N T R O D U C C I Ó N}

En Colombia, el Estado como garante de los derechos humanos no ha logrado satisfacer completamente la carga prestacional que envuelve el derecho al agua, especialmente frente a las comunidades campesinas o rurales. Ante tal ausencia estatal, en estas comunidades ha predominado la gestión comunitaria del agua, Así, se tiene que, históricamente, las comunidades ubicadas en las zonas rurales han organizado formas de gestión, administración y acceso al agua, para garantizar la satisfacción de sus necesidades básicas según sus usos, costumbres e identidad cultural.

Con la introducción en Colombia de la Ley 142 de 1994 se estableció el Régimen de Servicios Públicos Domiciliarios, que imprime una dinámica empresarial a la prestación del servicio de agua potable e incluye a las referidas comunidades dentro del conjunto de sujetos autorizados para el abastecimiento del recurso. De ello se derivan tensiones ampliamente documentadas, que amenazan la existencia de los acueductos comunitarios, entre la gestión comunitaria y el modelo de eficiencia económica, libertad de empresa y competencia contemplado en este régimen.

Por ende, el presente trabajo propone la implementación en Colombia de una política pública con enfoque de derechos humanos para la promoción y fortalecimiento de la gestión comunitaria del agua. Esto implica, según la definición de política pública con enfoque de derechos de la Comisión Interamericana de Derechos Humanos (CIDH, 2018), el diseño, implementación y evaluación de un programa de acción gubernamental con permanente participación social, a efectos de proteger, promover, respetar y garantizar los derechos humanos de 
personas, comunidades o sujetos de derechos colectivos. Así mismo, es necesario determinar los elementos jurídicos básicos sobre los cuales debe diseñarse tal política pública, a la luz de los desafíos que afronta la gestión comunitaria del agua de cara al régimen jurídico actual de servicios públicos domiciliarios.

\section{METODOLOGÍA}

Para la consecución de los referidos objetivos se utilizó una metodología hermenéutica y analítica de revisión de textos legales (fuentes primarias) y académicos (fuentes segundarias), con el objeto de establecer categorías básicas de análisis que orientaran el estudio de las implicaciones históricas y jurídicas en la gestión del agua (Salas, 2007; Sandoval, 2014). De igual forma, se apeló a la sociología de las ausencias desarrollada por De Sousa (2006) como procedimiento para hacer presentes una gran cantidad de conocimientos, saberes y experiencias antes ausentes o producidos como inexistentes por la razón metonímica y reduccionista del positivismo científico. Este procedimiento permitió reconocer la gestión comunitaria como una experiencia productiva relegada por la política pública estatal y el régimen jurídico de servicios públicos, que merece protección y promoción como una de las formas más puras de ejercer el derecho fundamental al agua.

Los resultados de la investigación se dividieron en dos grandes partes. En la primera de ellas, se pretende clarificar las categorías de gestión pública, privada y comunitaria del agua, a través de la exposición de experiencias normativas en Colombia con el objetivo de proporcionar un panorama sobre la gestión comunitaria del agua, su importancia y, en especial, las dificultades que enfrenta en el régimen jurídico actual. En la segunda parte, sobre la base de este diagnóstico, se exponen los aspectos jurídicos esenciales de una política con enfoque de derechos humanos para el fortalecimiento de la gestión del agua en las comunidades rurales o campesinas, a partir de la jurisprudencia constitucional y las disposiciones de instrumentos internacionales aplicables a esta materia, como una solución potencial, concreta y emergente frente a la homogenización de las prácticas productivas promovidas en la Ley 142 de 1994. 


\section{LA GESTIÓN DEL AGUA EN COLOMBIA: HACIA UNA COMPRENSIÓN DE LA GESTIÓN COMUNITARIA}

\section{La gestión del agua: aproximación conceptual, histórica y jurídica}

La gestión del agua puede caracterizarse de acuerdo con el sujeto encargado de la prestación del servicio, los objetivos que se persiguen y la forma en que se adoptan las decisiones (Sandoval y Günther, 2013). Históricamente, se conocen en esencia tres tipos de gestión del recurso hídrico, a saber: gestión pública, privada y comunitaria del agua con sus consecuentes implicaciones jurídicas y económicas.

En los Estados latinoamericanos el modelo de gestión del agua que se promovía y reconocía jurídicamente durante la mayor parte del siglo XX era esencialmente público. Esto significa que la gestión estaba a cargo del Estado. Según lo exponen Tobón y Valencia (2005), a partir de 1950, con la expedición del Decreto 259, en Colombia el Estado asume un papel preponderante en la prestación del servicio de agua, puesto que se le asignó al Instituto de Fomento Municipal (Insfopal), entidad pública del orden nacional, la ejecución de proyectos de alcantarillado, aseo y acueducto, a efectos de garantizar la universalidad en la prestación del servicio. Así mismo, se consolidaron las empresas municipales de Bogotá, Medellín y Cali, con la consecuente autorización a entidades territoriales para establecer empresas públicas encargadas del servicio.

Posteriormente, de acuerdo con los autores citados, la responsabilidad de la prestación del servicio se descentralizaría administrativamente de forma definitiva en 1986, trasladándose a las entidades territoriales del orden departamental y municipal, apoyados por los proyectos financieros de la Financiera de Desarrollo Territorial (Findeter). No obstante, las funciones de regulación, planeación y supervisión del sector se mantuvieron en cabeza del Gobierno nacional.

En contraste, la gestión privada del recurso hídrico se caracteriza porque la prestación del servicio se ajusta a un modelo de economía de mercado: el prestador es generalmente una empresa privada o una organización con tales características, es decir, un homo economicus (Cooter y Ulen, 2016) con una racionalidad tendiente a la maximización del beneficio social, a través de la satisfacción de las necesidades del usuario y la generación de utilidades. 
Este modelo de gestión empleado y desarrollado especialmente en Francia (Matés-Barco, 2013) fue el que existió en Colombia a finales del siglo XIX y las primeras décadas del siglo XX, cuando los municipios otorgaron concesiones a particulares para que desarrollaran y operaran infraestructuras que les permitieran abastecer de agua a la población con un margen de ganancia. Sin embargo, con la expedición de la Ley 65 de 1936 se produce una progresiva recuperación de esta función por parte del sector público a efectos de solucionar problemas en la universalidad del servicio, que tendrá como resultado un predominio del Estado, según lo expuesto anteriormente (Mesa et al., 2013).

No obstante, el modelo de gestión privada se promovió ampliamente después de 1980 en América Latina y Occidente en general debido a las recomendaciones de la comunidad internacional ${ }^{1}$, que a través de herramientas jurídicas de Softlaw como los Objetivos de Desarrollo del Milenio dieron lugar a estrategias de gobernanza, con el propósito de vincular la prestación del servicio público de agua y saneamiento básico a los particulares y grandes capitales privados para su prestación eficiente (Achkar y Domínguez, 2008).

De este modo, durante este periodo las expectativas estaban puestas en que la gestión privada con un adecuado marco regulatorio (libertad económica regulada), lograra resolver problemas estructurales como la falta de inversión en infraestructura, el escaso control en la calidad del agua y la desigualdad en el acceso al recurso de determinados grupos poblacionales (Zurbriggen, 2014). Este momento concluyó con reformas institucionales como la Ley 142 de 1994 en Colombia, que recoge en esencia los principios establecidos en la Constitución de 1991.

Así, este documento legal tiene como objetivo garantizar en términos de eficiencia paretiana la prestación del servicio público (Muñoz y Chía, 2012), en la medida en que promueve la libertad de entrada a este mercado y el control regulatorio de aquellas empresas que tengan una posición monopólica, para que la oferta de servicios aumente con una mejora en la calidad y a un mínimo costo para el usuario, a través de fórmulas tarifarias que den cuenta de la estructura de costos y aumentos de la productividad,

1 Por ejemplo, instituciones como el Banco Mundial y el Fondo Monetario Internacional a finales del siglo pasado y comienzos del nuevo milenio otorgaban préstamos a los Estados destinados al sector del agua potable y saneamiento básico, bajo la condición de que se promoviera la privatización del servicio. Al respecto puede consultarse Ávila et al. (2019). 
eviten el traslado de ineficiencias productivas al usuario y respondan al principio de solidaridad (Atehortúa, 2008).

Sin embargo, la gestión que se promueve normativamente no es exclusivamente privada. De conformidad con el artículo 5 de la Ley 142 de 1994, los municipios son las entidades encargadas de asegurar la prestación del servicio a los habitantes con el apoyo de la Nación y los Departamentos, de acuerdo con los principios de coordinación, concurrencia y subsidiariedad. Para ello, los municipios pueden prestar directamente el servicio en los supuestos previstos en el artículo 6 de la citada ley o a través de empresas de capital público, privado o mixto.

A pesar de que la historia de la regulación en Colombia se centra en las formas de gestión pública y privada del agua (Salamanca, 2001), existe también un modelo de gestión propio de la cultura latinoamericana en el que los usuarios se organizan para proveerse el servicio, generalmente a través de prácticas ancestrales que les permiten satisfacer sus necesidades básicas y de sobrevivencia, el cual se ha dado en llamar gestión comunitaria del agua.

Según lo expone Casas Cervantes (2015), en este modelo el recurso hídrico es gestionado desde la sociedad civil, razón por la cual las comunidades, especialmente en zonas rurales, organizan y administran el acceso, distribución y uso del agua a través de instituciones denominadas acueductos comunitarios. Estas instituciones funcionan sobre la base de valores y principios socioculturales compartidos por la comunidad, que se proyectan en las prácticas propias a través de las cuales se gestiona el recurso, como las tecnologías empleadas para la recolección y abastecimiento, los métodos de conservación del agua y los usos a los que se destina. Este fundamento cultural de los acueductos comunitarios los distingue de esquemas empresariales de explotación del recurso hídrico.

De este modo, la gestión comunitaria se ha caracterizado por (Defensoría del Pueblo, 2013):

1 La autogestión: a través de estructuras tradicionales que permiten a los actores locales desplegar un conjunto de acciones coordinadas para abastecerse del recurso de forma sostenible y de acuerdo con sus aspiraciones y expectativas culturales.

2 El trabajo solidario: los miembros de la comunidad se organizan en un marco de diálogo, participación y respeto por las diferencias para la toma de decisiones, generalmente a través de asambleas o juntas, en las que se discuten, proponen y aprueban planes orientados hacia la protección del recurso hídrico. 
3. La prestación del servicio ocurre principalmente en la ruralidad, en el seno de comunidades con arraigo territorial y sentido de pertenencia por sus acueductos comunitarios, a los que consideran como una parte esencial de ellos y frente a los cuales asumen responsabilidades y obligaciones para mantener sus funcionamiento (Linsalata, 2014).

En tal sentido, según lo expone Motta (2018) a través de la presentación de varias experiencias de gestión comunitaria del agua en Colombia, esta forma de gestión cumple un papel primordial en el Estado colombiano para garantizar el derecho fundamental al agua y asegurar condiciones de sostenibilidad ambiental en la administración del recurso, puesto que a través de los acueductos comunitarios de bocatomas y pequeñas infraestructuras se abastece y distribuye el agua, salvaguardando el ecosistema rural según lo usos y costumbres de las comunidades que ocupan estas zonas.

Con base en estas características, la gestión comunitaria del agua se ha definido como una actividad económica del sector solidario, en la que las comunidades organizadas por autorización del artículo 365 de la Constitución Política de 1991 se encargan de la prestación del servicio público domiciliario de agua potable, a través de acueductos comunitarios que reflejan una visión cultural particular sobre el territorio. Por lo tanto, dicha actividad responde a una concepción diferenciada del agua, que dista mucho de ser tratada como bien económico, y es abordada más bien como un elemento vital y esencial de las comunidades rurales (Boelens, 2009; 2011). A continuación, se analizará este modelo de gestión a la luz del régimen jurídico aplicable.

\section{La gestión comunitaria del agua en Colombia: avances y dificultades}

Las estrategias de gobernanza y la promoción de la libertad económica regulada para la provisión del servicio de agua potable, que representa el paradigma actualmente vigente en Colombia, de cierta manera han significado avances en el abastecimiento y saneamiento del agua. Cabe notar, sin embargo, que no han funcionado en otras latitudes del continente como Argentina, Bolivia y Uruguay, donde se llegó al extremo de prohibir la gestión privada del recurso desde el texto constitucional (Zurbriggen, 2014). De este modo, uno de los problemas estructurales que la participación del sector privado no ha logrado resolver en Latinoamérica es el abastecimiento del recurso hídrico en las poblaciones de las zonas rurales. 
La mayor parte de la población sin acceso al agua habita en estas zonas, pues la falta de congruencia en los planes implementados por las entidades gubernamentales con las condiciones socioeconómicas y culturales de estas poblaciones, sumada a la ineficiencia de los sistemas de control y mantenimiento por la falta de recursos técnicos y financieros, de acuerdo con Marin (2010), han minado la posibilidad real y efectiva de garantizar un acceso universal al agua potable bajo los estándares nacionales e internacionales.

Prueba de lo anterior es el informe de Naciones Unidas sobre "el estigma y el ejercicio de los derechos humanos al agua y al saneamiento" (ONU, 2011), que evidencia cómo ciertas poblaciones comúnmente ignoradas y marginadas tienen mayores dificultades para acceder a estos servicios. Analizando algunos de estos grupos de interés puede decirse, por ejemplo, que las comunidades indígenas se encuentran desproporcionadamente excluidas del acceso al agua en comparación con otras zonas urbanas, como también ocurre con múltiples comunidades campesinas y pueblos afrocolombianos (Bello y Rangel, 2002). Una realidad que ejemplifica la vulneración al principio de igualdad y no discriminación.

En este contexto de exclusión, la solución que han encontrado las comunidades para garantizar el consumo personal y doméstico del agua es la organización de acueductos comunitarios, debido a que ha sido a través de esta forma autóctona de gestión como estas han logrado satisfacer algunas necesidades inherentes a su calidad de vida, sin poner en riesgo el uso responsable y ambientalmente sostenible de los recursos naturales de los que disponen.

En este punto es importante resaltar la estrecha relación que se evidencia entre las comunidades organizadas en torno a los acueductos comunitarios y sus territorios tradicionalmente ocupados, así como el enfoque diferenciado que tienen respecto del aprovechamiento de las fuentes hídricas y los demás recursos naturales, bajo una racionalidad ambiental y cultural más que económica (Salazar, 2019). De este modo, la gestión comunitaria del agua envuelve en sí misma una ecología de las productividades, puesto que parte del reconocimiento del valor de los sistemas alternativos de producción, de las organizaciones económicas populares, de las empresas autogestionadas y de la economía solidaria (De Sousa, 2003).

Esta ecología de productividades reflejada en los acueductos comunitarios forma parte de la tradición histórica y cultural de las comunidades indígenas y campesinas en América Latina, donde se han identificado distintas formas organizativas comunitarias (Palerm y 
Martínez, 2009; Zurbriggen, 2011). Por ello, la gestión comunitaria tiene un rol doble: 1) representa una solución concreta al incumplimiento del principio de universalidad del servicio, y 2) constituye una tradición y práctica autóctona de las poblaciones rurales.

No obstante, uno de los principales desafíos que enfrenta la gestión comunitaria del agua es la falta de reconocimiento del derecho al agua como derecho fundamental ${ }^{2}$ y el deficiente apoyo institucional a esta forma de gestión, reflejado en las reducidas garantías jurídicas para la preservación de esta tradición (Casas, 2015). En ese sentido, según las evidencias que se exponen a continuación, en el caso colombiano esta forma de gestión del recurso ha sido una actividad descuidada por parte de la política pública estatal.

El reconocimiento jurídico de las comunidades organizadas en Colombia data del año 1962, a través del Programa de Saneamiento Básico Rural promocionado por el Gobierno. Dicho plan buscaba incentivar la autogestión en el sector para poblaciones de menos de 2500 habitantes, así como con población dispersa, con el fin de promover la construcción de acueductos en las pequeñas localidades con el apoyo de diferentes entidades (Hurtado et al., 2006).

Por otro lado, en la década del setenta la expedición del Decreto 2811 de 1974 otorgó reconocimiento y legitimidad a los gestores comunitarios de agua, permitiéndoles existir bajo la figura de las asociaciones de usuarios de agua. Con la promulgación del Decreto 1541 de 1978, se brindó prioridad a las concesiones de aprovechamiento de agua para el consumo humano, se organizaron las asociaciones de usuarios de agua y las denominadas empresas comunitarias para el aprovechamiento de las aguas y cauces, como medio de adquirir el derecho al uso de las aguas, concediendo a las comunidades del sector rural y de bajos recursos mayores garantías legales y organizativas para el autoabastecimiento de agua. Al mismo tiempo, se fortaleció el esquema organizativo destinado a las comunidades de bajos recursos que se quisieran estructurar como empresas comunitarias para provechar las aguas responsablemente, según lo dispuesto en el artículo 270 del mencionado decreto.

2 Contando los países de América Latina (18), sólo cinco reconocen el derecho al agua y saneamiento como un derecho humano fundamental de carácter constitucional; dos de ellos, reconocen el servicio y el agua como fundamentales, y los otros sólo reconocen el derecho al agua. Nueve de ellos mencionan el derecho al agua de alguna forma dentro de su constitución, y de todos ellos son Chile y Brasil los únicos que no poseen ningún tipo de norma o política que haga referencia al derecho humano al agua y al saneamiento (Mora y Dubois, 2015). 
La Carta Política de 1991 impregna el sistema económico nacional y la prestación del servicio de agua potable de una estructura neoliberal. Pese a ello, se reconocieron constitucionalmente, en el artículo 365, las comunidades organizadas como sujetos que pueden encargarse de la prestación del servicio. Concretamente, en desarrollo de esta disposición constitucional la Ley 142 y el Decreto 421 de 2000 establecen la forma en que las comunidades organizadas pueden ejercer la actividad de abastecimiento de agua potable en municipios menores, zonas rurales y áreas urbanas específicas, mediante el esquema de los servicios públicos domiciliarios.

A pesar de estos esfuerzos jurídicos de reconocimiento, en el Informe de Desarrollo Humano (ONU, 2006) se expuso que en Colombia existen diversas poblaciones que debido a su ubicación y su baja densidad poblacional no poseen un sistema de acueducto, como tampoco gozan del interés o inversión estatal para su desarrollo, ni poseen el nivel de organización jurídicamente exigido. De forma que las comentadas garantías jurídicas representan iniciativas insuficientes, como quiera que la intervención estatal ha permanecido al margen del proceso de desarrollo de estos acueductos, que hoy en día superan los 120.000 en el territorio nacional, algunos en poblaciones que no superan los 300 habitantes (Mesa et al., 2013).

Básicamente, en Colombia se ha transferido la carga regulatoria del régimen de servicios públicos domiciliarios a estas comunidades. De este modo, los gestores comunitarios para funcionar como prestadores del servicio deben constituirse como persona jurídica sin ánimo de lucro (Decreto 421, artículo 1), registrarse en la Cámara de Comercio, atender obligaciones tributarias, ser vigilados por la Superintendencia de Servicios Públicos Domiciliarios, estar sujetos a la regulación de la Comisión Reguladora de Agua Potable y Saneamiento Básico (CRA) (Decreto 421, artículo 3), y cumplir con los estándares de potabilización vigentes.

En el caso del Distrito Capital de Bogotá, el Decreto 552 de 2011 adoptó una serie de medidas con el objetivo de mejorar las condiciones de prestación de los servicios de agua potable y saneamiento básico por parte de comunidades organizadas en acueductos comunitarios. No obstante, esta normativa ha demostrado contener poca comprensión de las verdaderas necesidades de estas comunidades con escasos recursos y pocos conocimientos jurídicos y contables para implementar los requisitos contemplados en esta reglamentación. Esto debido a que el decreto dispone en su artículo 3 que las comunidades deben concurrir, aportar 
y/o facilitar la información y soportes tendientes a depurar la información contable de cada organización.

De esta forma, según lo expone Tascón (2018), la producción normativa y regulatoria en Colombia sobrepasa la capacidad operacional de la gran mayoría de gestores comunitarios de agua, como lo refleja también la Resolución 717 de 2015 de la CRA, que impone a las comunidades organizadas del sector rural un régimen tarifario con los componentes, fórmulas y variables que son aplicables a las empresas de servicios públicos. Lo que demuestra la intromisión arbitraria en los sistemas tradicionales y consuetudinarios de distribución del agua, esta regulación no tiene en cuenta la lógica comunitaria, ya que las formulas tarifarias sólo funcionan en esquemas organizativos de empresa (Tascón, 2018).

Así, ante la necesidad de enfoques diferenciados para las zonas rurales, el Plan Nacional de Desarrollo “Todos por un nuevo país” para el cuatrienio 2014-2018, dispuso en su artículo 18 que el Gobierno nacional definiera esquemas diferenciados para la prestación de los servicios de acueducto, aseo y alcantarillado. Por ello, en noviembre de 2016 el Gobierno dictó el Decreto 1898, que contempla condiciones diferenciales para los prestadores del servicio en la zona rural, y en el año 2017 expidió el Decreto 1272 sobre esquemas diferenciados en zonas del suelo urbano de difícil acceso, difícil gestión y condiciones particulares que impiden el cumplimiento de los estándares de eficiencia, cobertura y calidad.

De esta forma, en cuanto a la calidad del agua se dispone que el prestador establecerá un plazo de cumplimiento de los estándares de calidad con metas a corto y largo plazo, así como las precauciones y orientaciones técnicas para el consumo humano. Respecto a la micromedición, los prestadores podrán emplear procedimientos alternativos y realizar la facturación a partir de consumos estimados; y frente a la continuidad, el suministro podrá ser periódico siempre que se garanticen los volúmenes necesarios para satisfacer el consumo básico que fije la CRA. El Decreto 1898 establece que para el prestador acogerse a este esquema diferenciado, deberá formular un plan de gestión que satisfaga los requisitos dispuestos por el Ministerio de Vivienda, Ciudad y Territorio, y que contemple un plan de obras e inversiones en el que se indiquen los plazos en los que se ejecutarán los componentes de infraestructura para alcanzar los estándares de prestación del servicio, con el apoyo técnico del municipio.

Al respecto, la Red Nacional de Acueductos Comunitarios (RNAC, 2016) al analizar esta disposición normativa consideró que obliga a los acueductos comunitarios a adaptarse irremediablemente a formas propias 
del ámbito empresarial, como la formulación de planes de inversión y la capacidad financiera para llevarlos a término. En tal sentido, más allá de un esquema diferenciado esta regulación promueve la unificación de la prestación del servicio en términos de eficiencia económica por medio de un sistema de plazos. La referida unificación ha sido subrayada por la literatura como una premisa constante sobre la cual opera el actual régimen de servicios públicos, que ha sido promovida desde el Departamento Nacional de Planeación a través de la fusión de los acueductos comunitarios con empresas consolidadas de servicios públicos domiciliarios (Motta, 2018).

De esta manera, los acueductos comunitarios, sus imaginarios en cuanto al manejo del recurso hídrico, así como sus tradiciones y costumbres, se ven amenazadas por el poder regulador de la CRA que tiene la capacidad de fusionarlos con esquemas netamente empresariales de acuerdo con lo previsto en el artículo 73, numeral 14, de la Ley 142. Esta unificación se promueve progresivamente con los esquemas diferenciados citados, y amenaza "la continuidad de los acueductos comunitarios y recursos de las comunidades, limitando la gestión comunitaria del agua, en nombre del desarrollo empresarial del sector" (Bustos, 2014, p. 85).

No obstante, el decreto también contempla otra forma jurídica bajo la cual pueden operar los modelos comunitarios de gestión del agua, denominada soluciones alternativas para el aprovisionamiento de agua. Bajo esta modalidad el gestor pierde la calidad de prestador del servicio en los términos previstos por la Ley 142 de 1994, y se convierte en un administrador de un punto de abasto o suministro. Estas soluciones alternativas para el aprovisionamiento se caracterizan porque: 1) el acceso al agua para consumo humano podrá efectuarse mediante un abasto de agua o punto de suministro, directamente de la fuente según las necesidades de la comunidad y acorde a la normatividad; 2) el almacenamiento del recurso puede realizarse en tanques o dispositivos móviles; y 3) el tratamiento del líquido se hará mediante técnicas o dispositivos que lo permitan. En otras palabras, el proceso de abastecimiento se puede adelantar a través de los mecanismos tradicionalmente utilizados por los acueductos comunitarios.

Ahora bien, al perder la calidad de prestadores del servicio, el régimen de servicios públicos domiciliario contemplado en la Ley 142 deja de ser aplicable a estas soluciones alternativas, en la medida en que no se considera una actividad de prestación del servicio de agua. Por tal 
razón, estos administradores no están sujetos a la regulación de la CRA y tampoco son vigilados por la Superintendencia de Servicios Públicos Domiciliarios, simplemente cuentan con la supervisión de las autoridades sanitarias competentes.

Esto implica una reducción significativa de la carga regulatoria que debían asumir los acueductos comunitarios para operar legalmente, aunque siguen siendo aplicables para los administradores de estas soluciones las disposiciones concernientes al deber de constituirse como personas jurídicas sin ánimo de lucro y de obtener los permisos ambientales que les sean exigibles. Sobre este aspecto en particular, la RNAC (2016) ha considerado que el decreto

no reconoce que los acueductos puedan prestar sus servicios con agua cruda y los relega a la figura de administradores de soluciones alternativas para el acceso al agua. No puede así el decreto bajar de categoría a los acueductos comunitarios a simples administradores, desconociendo su papel histórico como prestadores del servicio bajo el argumento del no cumplimiento de los índices de potabilidad. (pp. 4-5; nuestro énfasis)

Así las cosas, si bien el Gobierno Nacional a través de los comentados decretos pretende dar un tratamiento jurídico diferenciado a la gestión del agua en las zonas rurales y algunas áreas del suelo urbano, para progresivamente alcanzar metas de eficiencia y cobertura, termina generando un problema de identidad y reconocimiento frente a actores sociales relevantes que se consideran a sí mismos, y con amparo en la Carta Política, como verdaderos prestadores del servicio de agua.

De ahí la necesidad de una política pública integral con enfoque de derechos humanos que realmente promueva la gestión comunitaria del agua, sin desmontar los avances en cobertura alcanzados a través del régimen de servicios públicos domiciliarios. Esto es, una solución que promueva la igualad, equidad y pluralismo en la gestión del recurso hídrico, en lugar de una monocultura del productivismo, en la que predomina "la idea de que el crecimiento económico y la productividad mensurada en un ciclo de producción determinan la productividad del trabajo humano o de la naturaleza, y todo lo demás no cuenta” (De Sousa, 2006, p. 25). 


\section{LA NEGESIDAD DE UNA POLÍTICA CON ENFOQUE DE DERECHOS HUMANOS FRENTE A LA GESTIÓN COMUNITARIA DEL AGUA EN COLOMBIA}

Según lo expuesto, la política pública en el sector rural ha sido precaria en incentivos reales que promuevan la gestión comunitaria del agua y el desarrollo de estas economías rurales, dado que, como lo reconoció la Corte Constitucional en Sentencia C-644 de 2012, la población campesina sigue siendo la más pobre del país y la que vive en las condiciones de mayor vulnerabilidad.

Por lo tanto, esto amerita que se piense el sector rural desde los derechos humanos, esto es, desde el conjunto de garantías, obligaciones y deberes que se desprenden para el Estado colombiano de acuerdo con los instrumentos jurídicos nacionales e internacionales de derechos humanos que lo vinculan; especialmente frente a la prestación del servicio público de agua, que constituye un servicio meritorio, dada su calidad de bien público indispensable para la eficacia real del principio de dignidad humana (Sen, 1998).

Así, es necesario el desarrollo y adopción de una política pública para la gestión comunitaria del agua con enfoque de derechos humanos, que recoja las peculiaridades de cada región y de la ruralidad, esto es, que parta del reconocimiento del otro que está en la periferia en términos de Dussel (2007).

Las políticas públicas con enfoque de derechos envuelven un conjunto de decisiones y acciones que el Estado diseña, implementa y evalúa, en el marco de un proceso de diálogo y participación social, en el que son consultados los distintos sectores de interés (Abramovich, 2006a), con el objetivo de respetar y garantizar los derechos humanos de las personas, atendiendo los principios de igualdad y no discriminación, universalidad, acceso a la justicia, rendición de cuentas, transparencia, transversalidad e intersectorialidad, según lo expone la CIDH (2018).

Por ende, según Abramovich (2006b), las políticas con enfoque en los derechos humanos parten de una base axiológica como lo es el reconocimiento de derechos en cabeza de personas, grupos o colectividades. De forma que, más que una consideración ontológica de estos como sujetos con necesidades, se los concibe como sujetos de derecho, con la facultad de exigir o demandar de la institucionalidad el cumplimiento de prestaciones concretas. En consecuencia, este enfoque 
propicia el empoderamiento e inclusión de grupos tradicionalmente marginados, discriminados o desprotegidos, que se convierten en titulares de derechos con el poder de reivindicar cambios en el tratamiento jurídico, político y económico que reciben del Estado y de los particulares, que den lugar a avances estructurales en el goce de sus derechos (GómezGalán et al., 2013).

Por lo tanto, esta política debe partir del reconocimiento al derecho al agua, como derecho fundamental que envuelve obligaciones prestacionales positivas y negativas a cargo del Estado, así como obligaciones en cuanto a la conservación de formas culturales y tradicionales de gestión del recurso hídrico para el consumo humano. Ello implica el empoderamiento de las comunidades rurales y de los acueductos comunitarios, a través de la protección y promoción de los elementos de autogestión, trabajo solidario y ruralidad de la gestión comunitaria del agua.

La Corte Constitucional colombiana en Sentencia T-418 de 2010, con fundamento en el referido enfoque, ha señalado que una política pública frente al derecho fundamental al agua debe cumplir unas condiciones básicas, a saber: constituir un programa de acción estructurado que dé lugar a medidas adecuadas, necesarias y proporcionales; tener como prioridad garantizar el goce efectivo de este derecho de acuerdo con los estándares establecidos, atendiendo los verdaderos problemas y necesidades de los titulares del derecho; y finalmente, que los procesos de decisión, elaboración, ejecución y evaluación se den bajo el principio de participación.

A continuación se analizan los aspectos fundamentales que deben caracterizar una política pública que reconozca y promueva de forma diferenciada la gestión comunitaria del agua, fundada en el enfoque de derechos humanos propuesto sobre la base de las reflexiones de Víctor Abramovich.

\section{Reconocimiento del derecho al agua como fundamental y su centralidad en la gestión comunitaria del agua}

El agua ha sido entendida como un derecho humano fundamental tanto en el ordenamiento jurídico colombiano como en el derecho internacional, especialmente en la labor de Naciones Unidas respecto de la Observación General N. ${ }^{\circ} 15$ del Comité de Derechos 
Económicos Sociales y Culturales, y de la Resolución 64/292 de 2010³ de la Asamblea General de Naciones Unidas.

Las propuestas teóricas de autores como Urquhart y Mesquita (2014), Ramírez y Benítez (2016) y, principalmente, de Arrojo (2005), coinciden en señalar el acceso al agua para consumo personal o doméstico como un derecho de fundamento natural, derivado de la misma condición humana que requiere del agua como líquido para vivir, lo que la asimila al concepto de bien fundamental ${ }^{4}$ de Ferrajoli, inherente a la existencia humana bajo condiciones dignas.

En sentido similar, la Corte Constitucional en providencia T-740 de 2011 ha realizado un bosquejo general del contenido teórico del derecho al agua, al señalar que "se erige como una necesidad básica, al ser un elemento indisoluble para la existencia del ser humano", que se caracteriza por ser una necesidad universal, inalterable y objetivamente reconocible. Por esta razón, se erige como una necesidad normativa, esto es, que tiende un puente entre el ser y el deber ser dada la pretensión jurídica universal de garantizar el acceso al agua a todas las personas sin discriminación alguna.

Reconocer la centralidad del derecho fundamental al agua en la política pública que promueva la gestión comunitaria es indispensable, puesto que si bien esta es una actividad económica asociada al sector solidario, los acueductos comunitarios no son propiamente el resultado del ejercicio de las libertades económicas previstas en nuestra Constitución Política, en virtud de las cuales se constituyen empresas con fines de generar utilidades y competir en el mercado. Los acueductos comunitarios en cambio representan el ejercicio más puro del derecho fundamental al

3 Previamente había sido tratada implícita o explícitamente como derecho en instrumentos como la Conferencia Internacional sobre Agua y Desarrollo Sostenible, también conocida como la Conferencia de Dublín de 1992; la Conferencia de las Naciones Unidas sobre el Medio Ambiente y el Desarrollo o Cumbre de Río 1992, en el capítulo 18 del programa; la Conferencia Internacional de las Naciones Unidas sobre la Población y el Desarrollo de 1994; el programa Hábitat de 1996; la resolución 54/175 de la Asamblea General de las Naciones Unidas de 1999, relativa al derecho al desarrollo; la resolución 55/196 de la Asamblea General de las Naciones Unidas de 2000, que proclamó 2003 como Año Internacional del Agua Dulce; la Cumbre Mundial sobre el Desarrollo Sostenible de 2002; la resolución 58/217 de la Asamblea General de las Naciones Unidas en 2003, que declaró el Decenio Internacional para la Acción "El agua, fuente de vida"; la resolución 59/228 de la Asamblea General de las Naciones Unidas de 2004; el proyecto de directrices para la realización del derecho al agua potable y al saneamiento E/CN.4/Sub.2/2005/25, contenido en el informe del relator especial para el Consejo Económico y Social de las Naciones Unidas en 2005; y la decisión 2/104 del Consejo de Derechos humanos sobre los derechos humanos y el acceso al agua de 2006

4 Bien fundamental, contrapuesto al concepto de bien patrimonial, igualmente abordado por el jurista italiano, hace referencia a aquellos intereses cuya accesibilidad se garantiza de forma generalizada al estar íntimamente relacionados con otros derechos fundamentales. En ese sentido, son bienes que escapan a la lógica del mercado, y que se sustraen de una concepción basada en los intercambios voluntarios, lo que a su vez les otorga la categoría de indisponible (Contreras, 2012). 
agua, en la medida en que los mismos usuarios que requieren el líquido para su consumo personal y doméstico se autoabastecen a través de una acción organizada con base en sus usos y tradiciones. La persona es aquí un ser responsable de sí mismo y de la comunidad.

De modo que la protección jurídica de esta forma de gestión del agua no procede de la libertad de empresa y competencia ni del principio de intervención del Estado en la economía, como ocurre con las empresas públicas, privadas o mixtas de servicios públicos, sino del derecho natural a acceder al recurso hídrico para proveerse de un medio indispensable para la vida. Este es el sustento iusfilosófico de la gestión comunitaria del agua. Esta distinción entre el fundamento axiológico de las formas de gestión del agua demanda la existencia de un tratamiento jurídico diferenciado para la gestión comunitaria. Razón por la cual se debe promover la formalización de las comunidades rurales y campesinas gestoras del agua bajo modelos asociativos que reconozcan sus particularidades históricas, culturales y sociales, así como su relación con el territorio (Casas, 2015; Ostrom, 1990).

De la misma manera, también es necesario que se establezcan requisitos diferenciales para la concesión de la autorización del uso de cuencas hídricas, a efectos de que los acueductos comunitarios operen legítimamente y exista un registro ajustado a la realidad de estas formas de gestión, administrado por autoridades ambientales articuladas con las instancias de gobierno territorial (municipios y departamentos).

\section{Obligaciones del Estado frente a la gestión comunitaria del agua sobre la base del reconocimiento del derecho fundamental al agua}

Desde la perspectiva internacional, en la Observación General N. ${ }^{\circ} 15$ de la $\mathrm{ONU}^{5}$ sobre el derecho al agua, instrumento que desarrolla extensamente

\footnotetext{
5 La Observación General N. ${ }^{\circ} 15$ no es un texto vinculante para el Estado colombiano de manera directa, pero se vuelve esperanzador para la Gestión Comunitaria del Agua gracias a la jurisprudencia de la Corte Constitucional, al hacer una inclusión del contenido de dicha Observación como criterio hermenéutico determinante para la interpretación y aplicación autorizada del Pacto Internacional de Derechos Económicos, Sociales y Culturales (tratado internacional ratificado), introduciendo el Derecho al Agua para consumo humano como un Derecho Fundamental dentro del Bloque de Constitucionalidad, ya que anteriormente solo se hacía un ejercicio de protección por conexidad con los derechos a la vida, a la salud y a la dignidad humana. Esta nueva interpretación del derecho al agua es una evolución que otorga dimensiones más grandes al tratamiento que la Corte Constitucional ya le daba, y se puede evidenciar en la sentencia T-578 de 1992, y en las proferidas con posterioridad a la Observación General N. ${ }^{\circ} 15$, T-616 de 2010, T-740 de 2011, T-131 de 2016, entre otras.
} 
la materia, se evidencia la obligación que tienen los Estados de respetar, proteger y cumplir el derecho de toda persona al agua potable (Burgos, 2019). Con relación a la obligación de respeto, esta envuelve el deber de los Estados de cumplir directamente con los estándares de disponibilidad, accesibilidad y continuidad del líquido ${ }^{6}$, ya sea absteniéndose de actuar o dando una prestación (Nash, 2009), de manera que aseguren su aplicación a todos los individuos del territorio sometidos a su jurisdicción, en condiciones de igualdad y no discriminación (Gross Espiell, 1991; ONU, 2004).

En el caso de las comunidades organizadas para la prestación del servicio de agua, la única forma en la que se le permitiría al Estado actuar de conformidad con sus obligaciones es precisamente ajustándose a este deber, absteniéndose de intervenir arbitrariamente en la gestión comunitaria del agua, pues debe privilegiarse la forma tradicional de administración del recurso hídrico. No obstante, el Estado puede efectuar consultas con las comunidades para la implementación de proyectos de cooperación, siempre que la intervención estatal no genere un traumatismo en estas comunidades, sino por el contrario fortalezca sus tradiciones a través de un diálogo que desarrolle el principio de participación y permita la comprensión de sus necesidades, usos y costumbres (Abramovich, 2006b), con miras a proteger sus formas propias de abastecimiento del agua.

Este aspecto debe ser observado en la política pública a fortiori, porque la Corte Constitucional ha manifestado que el contenido de esta obligación de respeto incluye el deber de no inmiscuirse arbitrariamente en los sistemas consuetudinarios o tradicionales de distribución del agua ${ }^{7}$. De esta forma, las políticas regulatorias tendientes a la unificación de estas formas de gestión comunitaria con las dinámicas empresariales expuestas, constituyen una vulneración a la obligación de respeto del derecho al agua con relación al estándar de disponibilidad del líquido (García, 2017).

\footnotetext{
6 Los estándares obligatorios se pueden definir así: 1) disponibilidad: existencia del recurso de manera continua y suficiente; 2) accesibilidad: física, económica y sin ningún tipo de discriminación; 3) calidad, como la garantía de que el recurso se esté suministrando de manera salubre y no perjudicial para la salud humana (ONU, 2002); 4) asequible para todas las personas, lo que significa que los estados deben considerar todas las condiciones de acceso, incluidas las tarifas, los gastos y el almacenamiento de agua; 5) por último la aceptabilidad, en lo que respecta a su sabor, color y olor (Heller, 2015).

7 Al respecto pueden contarse numerosas experiencias de acceso y distribución tradicional del agua que son respetadas por las legislaciones nacionales, tales como los jueces del agua en Venezuela en la zona de Quibor, los comités de agua potable en Bolivia y las Juntas del Agua en Centroamérica.
} 
Por esta razón, la facultad de la CRA de fusionar prestadores del servicio debe ser restringida en una política de enfoque diferencial y de derechos, que reconozca que las formas de gestión pública, privada y comunitaria pueden coexistir bajo un marco de horizontalidad, en el que se respeten los valores tradicionales de las comunidades rurales sin imponerle lógicas o racionalidades económicas extrañas para ellas, salvo que estas deseen, en virtud de su autonomía, dar este paso, el cual debe estar apoyado por el Estado a través de asesorías técnicas y apoyo financiero.

El respeto a las prácticas de gestión comunitaria y su promoción a través de un diálogo abierto que busque inteligibilidad entre saberes y prácticas, en lugar de su homogeneización en torno a esquemas empresariales (De Sousa, 2006), es la mejor manera de promover la obligación estatal de cumplir con relación al estándar de disponibilidad, puesto que así se facilita el acceso al agua en la zonas rurales y las zonas urbanas desfavorecidas. Esto debe acompañarse de una vigilancia estatal diferencial para los acueductos comunitarios que promueva los estándares internacionales de accesibilidad y calidad, y fomente un uso ajustado a la cosmovisión de la comunidad pero seguro para la vida, salud e integridad de sus miembros ${ }^{8}$.

\section{Reconocimiento de las comunidades campesinas y rurales como sujetos colectivos de derecho}

Finalmente, un paso transcendental es que la política pública reconozca a las comunidades campesinas o rurales como sujetos colectivos de derechos, aunque no estén formalizadas. Los pueblos indígenas, campesinos y en general las comunidades de zonas rurales conciben el agua como un bien comunitario y un recurso indisociable de la tierra (Arrojo, 2009). De hecho, para suministrar el agua los pueblos indígenas andinos realizan desde tiempos inmemoriales gestiones comunitarias a través de mecanismos de reciprocidad, que generan vínculos reconocidos como la base de la subsistencia de sus miembros y su cultura (Ortiz, 2008).

\footnotetext{
$8 \mathrm{Al}$ respecto pueden verse las iniciativas de la Fundación Bill y Melinda Gates en África, que sin desconocer los valores comunitarios de las poblaciones, han fomentado el uso de soluciones tecnológicas accesibles para prevenir las muertes y enfermedades que se producen como consecuencia de prácticas sanitarias riesgosas, especialmente para los niños, niñas y adolescentes; por ejemplo, que por los mismos canales de abastecimiento del recurso hídrico para consumo humano, se desechen o dispongan las heces y residuos sólidos o líquidos.
} 
Por lo tanto, es evidente que las comunidades rurales cuentan con rasgos distintivos de la población ordinaria, especialmente de aquellas personas que viven en los principales centros urbanos. Estos rasgos distintivos si bien no han sido necesariamente reconocidos por los ordenamientos jurídicos en el derecho comparado o internacional, son muy similares a los rasgos que distinguen a las comunidades tribales o semitribales a partir del Convenio 169 de la OIT, lo que ha dado pie para un estatus jurídico especial, así como una protección internacional específica.

En otras palabras, la similitud en los aspectos fácticos de la protección de las comunidades indígenas y las comunidades campesinas, hace semejable la ratio legis que les permite gozar de una categoría específica de protección, dada la relación especial que ostentan dichas comunidades con su territorio, sus recursos naturales y la gestión responsable de los mismos (López-Murcia y Maldonado-Colmenares, 2009). Al analizar a las comunidades campesinas resulta evidente que las mismas cuentan con un apego especial a la tierra y una relación diferente con el territorio, y que entienden el agua como parte de este concepto de territorio.

Así mismo, su organización comunitaria es distinta a la organización tradicional, pues se rige por elementos tradicionales de su cultura campesina, diferente a los elementos que rigen las organizaciones formales como las sociedades comerciales. Como también lo dispone la Declaración de los derechos de los campesinos y de otras personas que trabajan en zonas rurales (ONU, 2013), al reconocer que campesino es "un hombre o una mujer de la tierra, que tiene una relación directa y especial con la tierra y la naturaleza a través de la producción de alimentos u otros productos agrícolas".

Es importante resaltar, como lo hace el Convenio sobre diversidad biológica (ONU, 1992), que la protección de estas formas ancestrales de conservación y explotación garantizan la utilización sostenible de los recursos naturales, esto significa que generalmente las prácticas comunitarias están impregnadas de una racionalidad ambiental de paz con la naturaleza. En consecuencia, el diseño de una política pública con enfoque de derechos debe ser un proceso participativo que tenga en cuenta que las comunidades campesinas y rurales son sujetos de especial protección, en atención a su arraigo con el territorio y a sus tradiciones (Abramovich, 2006b).

De manera que debe estructurarse un proceso de consulta por niveles dentro de los Departamentos, establecerse comités promotores con reuniones regionales y, posteriormente, llevar a una gran asamblea las 
propuestas de reglamentación que serán aprobadas, con la participación de organismos internacionales como garantes del proceso.

En la fase de implementación deberá establecerse un proceso de diagnóstico nacional de las comunidades campesinas en términos de calidad del agua, para definir el proceso de mejora de la accesibilidad a este recurso y los niveles de calidad con los cuales llega a los hogares de los campesinos, basado ante todo en un enfoque diferencial que reconozca que en lo rural se requiere un estándar de agua segura.

La participación de estas comunidades en el desarrollo e implementación de la política pública es esencial para evitar y resolver conflictos territoriales que se hayan originado como consecuencia de la ausencia de diálogo y compromiso del Estado con relación a la gestión comunitaria del agua (Díaz y Gómez, 2019). Así mismo, la política en sí misma debe procurar evitar ser una fuente de nuevos conflictos en los territorios de estas comunidades. Por lo cual es necesario manejar una articulación y responsabilidad institucional que propicie la rendición de cuentas y en especial los escenarios de solución de conflictos con agentes públicos o privados.

\section{CONGLUSIONES}

El enfoque de derechos humanos puede proveer respuestas que permitan equilibrar la protección de los intereses económicos de las empresas tradicionales, con los motivos de protección cultural y social de las formas comunitarias de gestión de agua, de manera que se resuelva, a través de una política pública diferencial, la tensión existente entre la racionalidad económica del régimen de servicios públicos domiciliarios y la racionalidad ambiental y cultural de los gestores comunitarios del agua. Esto con el fin de propiciar una ecología de las productividades en la que no se desconozcan las prácticas productivas de las comunidades rurales en torno al acceso al agua.

Lo anterior implica que el Estado debe respetar y proteger las formas asociativas tradicionales y las formas de acceso al agua de estas comunidades. En ese sentido, cualquier problema en la prestación del servicio debe ser suplido por el Estado apoyando y no reemplazando a los acueductos comunitarios, a través de una política pública que parta del reconocimiento del derecho fundamental al agua y de estas comunidades como sujetos colectivos de especial protección. 
Todo ello, con el fin de que se consiga la expedición de una ley estatutaria sobre el derecho fundamental al agua y la promoción de la gestión comunitaria después de consultar a las comunidades, que tenga como ejes centrales: 1) el desarrollo de requisitos diferenciales para la concesión de la autorización del uso de las cuencas hídricas; 2) el establecimiento de controles diferenciales en materia de calidad de agua y de usos y distribución del agua; 3) la definición de órganos competentes para la vigilancia y control de los gestores comunitarios del agua; y 4) la definición de los requisitos para la constitución de las organizaciones gestoras del agua.

\section{REFERENCIAS}

Abramovich, V. (2006a). Los estándares interamericanos de derechos humanos como marco para la formulación y el control de las políticas sociales. Anuario de derechos humanos, 2, 13. https://doi.org/10.5354/0718-2279.2006.13370

Abramovich, V. (2006b). Una aproximación al enfoque de derechos en las estrategias y políticas de desarrollo. Revista de la CEPAL, 88, 35-50. https:// doi.org/10.18356/a48f3cca-es

Achkar, M. y Domínguez, A. (2008). La gestión del agua desde la geopolítica trasnacional y desde los territorios de la integración. En D. Soares, S. Vargas y M.R. Nuño (Eds.), La gestión de los recursos hidricos: realidades y perspectivas (pp. 27-56). Universidad de Guadalajara.

Arrojo, P. (2005). Las funciones del agua: Valores, derechos, prioridades y modelos de gestión. En P. Arrojo et al. (Eds.), Lo público y lo privado en la gestión del agua. Experiencias y reflexiones para el siglo XXI (pp. 17-36). Ediciones del Oriente y del Mediterráneo; Fundación Alternativa.

Arrojo, P. (2009). Tipología y raíces de los conflictos por el agua en el mundo. En Fundación Seminario de Investigación Para la Paz (Ed.), El agua, derecho humano y raíz de conflictos (pp. 103-128). Gobierno de Aragón.

Atehortúa Ríos, C. A. (2008). Régimen de los servicios públicos domiciliarios: en el contexto de la doctrina constitucional. Diké.

Ávila, E., Merino, V. y Ortega, P. (2019). Manual de remunicipalización de los servicios públicos de agua. Aeopas.

Bello, M. y Rangel, M. (2002). La equidad y la exclusión de los pueblos indígenas y afrodescendientes en América Latina y el Caribe. Revista de la CEPAL, 76, 39-54. https://doi.org/10.18356/61fc0d54-es

Boelens, R. (2009). Aguas diversas. Derechos de agua y pluralidad legal en las comunidades andinas. Anuario de Estudios Americanos, 66(2), 25-55. http:// biblioteca.ues.edu.sv/revistas/10702571.1.pdf https://doi.org/10.3989/ aeamer.2009.v66.i2.316

Boelens, R. (2011). Luchas y defensas escondidas. Pluralismo legal y cultural como una práctica de resistencia creativa en la gestión local del agua en los 
Andes. Anuario de Estudios Americanos, 68(2), 673-703. http://www.cedla.uva. nl/20_research/pdf/Boelens/Boelens_AnuarioEstAm2011.pdf https://doi. org/10.3989/aeamer.2011.v68.i2.554

Burgos Cárdenas, V. M. (2019). Conceptualización sobre la responsabilidad patrimonial del Estado colombiano por la existencia de un Estado de cosas inconstitucionalECI frente al derecho fundamental al agua. http://94.23.83.42:8080/ bitstream/001/2198/1/GOAAA-spa-2019-Conceptualizacion_sobre_la_ responsabilidad_patrimonial_del_Estado_colombiano_por_la_existencia_ de_un_Estado_de_cosas

Bustos, I. J. G. (2014). La acción colectiva del agua en Colombia y el referendo como acercamiento de democracia directa. Análisis político, 27(80), 79-103. https://doi.org/10.15446/anpol.v27n80.45615

Casas Cervantes, A. F. (2015). La gestión comunitaria del agua y su relación con las políticas públicas municipales: el caso del manantial de Patamburapio en el estado de Michoacán, 2009-2014. Intersticios sociales, 10, 1-43.

Comisión Interamericana de Derechos Humanos. (2018). Políticas Públicas con enfoque de derechos humanos. http://www.oas.org/es/cidh/informes/pdfs/ PoliticasPublicasDDHH.pdf

Constitución Política de la República de Colombia. (1991, julio 6). Gaceta Constitucional, N. ${ }^{\circ} 116,1991$, julio 20.

Contreras, S. (2012). Ferrajoli y su teoría de los Derechos Fundamentales. Estudios de Filosofía Práctica e Historia de las Ideas, 14(2), 17-28.

Convenio sobre pueblos indígenas y tribales (C169). (1989, junio 27). https:// www.ilo.org/wcmsp5/groups/public/---americas/---ro-lima/documents/ publication/wcms_345065.pdf

Cooter, R. y Ulen, T. (2016). Derecho y economía. Fondo de Cultura Económica.

Corte Constitucional Colombiana. (2010). Sentencia T-418 (María Victoria Calle, M.S.).

Corte Constitucional Colombiana. (2011). Sentencia T-740 (Humberto Antonio Sierra, M.S.).

Corte Constitucional Colombiana. (2012). Sentencia C-644 (Adriana María Guillén, M.S.).

De Sousa Santos, B. (2003). Crítica de la razón indolente: contra el desperdicio de la experiencia (Vol. 1). Desclée de Brouwer.

De Sousa Santos, B. (2006). Renovar la teoría crítica y reinventar la emancipación social. Clacso.

Decreto 1272 del 28 de julio de 2017. (2017, julio 28). Diario Oficial, N. ${ }^{\circ} 50.308$.

Decreto 1898 del 23 de noviembre de 2016. (2016, noviembre 23). Diario Oficial, N. ${ }^{\circ}$ 50.066 .

Decreto 2811 del 18 de diciembre de 1974 (Código Nacional de Recursos Naturales Renovables y de Protección al Medio Ambiente). (1975, enero 27). Diario Oficial, N. ${ }^{\circ} 34.243$.

Decreto 421 del 8 de marzo de 2000. (2000, marzo 13). Diario oficial, N. ${ }^{\circ} 43.932$.

Decreto 552 del 6 de diciembre de 2011. (2011, diciembre 7). Registro Distrital de Bogotá, N. ${ }^{\circ} 47788$. 
Decreto por el cual se reglamenta la Parte III del Libro II del Decreto-Ley 2811 de 1974. (Decreto 1541). (1978, julio 26).

Defensoría del Pueblo. (2013). Gestión Comunitaria del Agua. https://www. defensoria.gov.co/es/public/Informesdefensoriales/733/La-gesti\%C3\%B3ncomunitaria-del-agua-Informes-defensoriales---Agua.htm

Díaz, O. H. y Gómez, D. L. (2019). Las políticas rurales y su incidencia en la formación de conflictos territoriales. Prolegómenos, 22(43), 43-62. https://doi. org/10.18359/prole. 3434

Dussel, E. (2007). La filosofía de la liberación ante el debate de la posmodenidad y los estudios latinoamericanos. Jáuregui y Moraña. Colonialidad y crítica en América Latina. Universidad de las Américas.

García Pachón, M. (2017). Régimen Jurídico de los Vertimientos en Colombia. Universidad Externado de Colombia.

Gómez-Galán, M., Pavón, D. y Sainz, H. (2013). El enfoque basado en derechos humanos en los programas y proyectos de desarrollo. Cideal.

Gross Espiell, H. (1991). La convención americana y la convención europea de Derechos Humanos: Análisis comparativo. Ed. Jurídica de Chile

Heller, L. (2015). El derecho humano al agua potable y el saneamiento. Informe del Relator Especial sobre el derecho humano al agua potable y el saneamiento Consejo de Derechos Humanos. Asamblea General de la ONU.

Hurtado, H., Suescún, S., Toro, R., Valencia, N., Monroy, J., Henao, O. y Bedoya, I. (2006). Servicios públicos domiciliarios: Seis aplicaciones en el caso del departamento de Antioquia. Universidad de San Buenaventura.

Linsalata, L. (2014). Ni público, ni privado: común. Prácticas y sentidos de la gestión comunitaria del agua en la zona sur de Cochabamba en Bolivia. Territorios en disputa, 249.

López-Murcia, J. D. y Maldonado-Colmenares, G. (2009). La protección de la propiedad de la tierra en la jurisprudencia de la Corte Interamericana de Derechos Humanos y su aplicación al caso de las comunidades campesinas en Colombia. International Law: Revista Colombiana de Derecho Internacional, 14, 71-105.

Marín, G. (2010). Coberturas, acceso al abastecimiento y saneamiento. En Ingeniería Sin Fronteras Asociación para el Desarrollo (Ed.), Derecho al agua, implementación del derecho humano al agua (pp. 18-25). Unesco Etxea.

Matés-Barco, J. M. (2013). La conquista del agua en Europa: Los modelos de gestión (siglos XIX y XX). Agua y territorio, 1, 21-29. https://doi.org/10.17561/ at.vli1.1030

Mesa, J. M., Muñoz, C. P. y Agudelo, G. D. V. (2013). Comunidades organizadas y el servicio público de agua potable en Colombia: Una defensa de la tercera opción económica desde la teoría de recursos de uso común. Ecos de economía, 17(37), 125-159. https://doi.org/10.17230/ecos.2013.37.6

Mora, J. y Dubois, V. (2015). Implementación del derecho humano al agua en América Latina (Informe técnico). https://scioteca.caf.com/handle/123456789/789 
Motta, R. (2018). El derecho de la gestión comunitaria del agua en Colombia para garantizar el acceso al agua potable. Misión Jurídica, 11(15), 321-334.

Muñoz, M. D. P. S. y Chía, M. I. U. (2012). Economía de los servicios públicos domiciliarios, más allá del mercado y del Estado. Equidad Ẽ Desarrollo, 17, 3156. https://doi.org/10.19052/ed.66

Nash, C. (2009). El Sistema Interamericano de Derechos humanos en acción. Aciertos y desafíos. Editorial Porrúa.

ONU. (1992). Convenio sobre biodiversidad biológica. https://www.cbd.int/doc/legal/ cbd-es.pdf

ONU. (2002). Observación general N. ${ }^{\circ}$ 15: El derecho al agua (artículos 11 y 12 del Pacto Internacional de Derechos Económicos, Sociales y Culturales). Ginebra (Suiza).

ONU. (2004). Observación General N. ${ }^{\circ} 31$. Naciones Unidas, Comité de Derechos Humanos. Doc. HRI/GEN/1/Rev.7 at 225.

ONU. (2006). Informe de Desarrollo Humano 2006 - Más allá de la escasez: poder, pobreza y la crisis mundial del agua, del Programa de las Naciones Unidas para el Desarrollo. http://hdr.undp.org/sites/default/files/hdr_2006_es_completo.pdf

ONU. (2011). Informe de la Relatora Especial sobre el derecho humano al agua potable y el saneamiento, Catarina de Albuquerque. https://documents-dds-ny.un.org/doc/ UNDOC/GEN/G11/153/82/PDF/G1115382.pdf?OpenElement

ONU. (2013). Declaración sobre los derechos de campesinos y otras personas que trabajan en zonas rurales. https://www.ohchr.org/Documents/HRBodies/HRCouncil/ WGPleasants/A-HRC-WG-15-1-2_sp.pdf

Ortiz, A. (2008, mayo 28). La legislación hidrológica orientada al libre mercado como modelo de reformas en los países andinos: planteamiento del problema. Revista Cuadernos de desarrollo rural, 5, 87-111.

Ostrom, E. (1990). Governing the commons: The evolution of institutions for collective action. Cambridge University Press. https://doi.org/10.1017/ CBO9780511807763

Palerm, J. y Martínez, T. (2009). Aventuras con el agua. La administración del agua de riego: historia y teoría. Colegio de postgraduados.

Plan Nacional de Desarrollo 2014-1018 (Ley 1753 del 9 de junio de 2015). (2015, junio 9). Diario Oficial, N. ${ }^{4} 4538$.

Ramírez, J. D. J. B. y Benítez, I. S. (2016). El derecho humano al acceso al agua potable: aspectos filosóficos y constitucionales de su configuración y garantía en Latinoamérica. Revista Prolegómenos. Derechos y Valores de la Facultad de Derecho, 19(37), 125-146. https://doi.org/10.18359/prole.1684

Red Nacional de Acueductos Comunitarios. (2016). Observaciones al proyecto de decreto: "Por el cual se reglamenta parcialmente el artículo 18 de la Ley 1753 de 2015, en lo referente a esquemas diferenciales para la prestación de los servicios de acueducto, alcantarillado y aseo en zonas rurales". http://redacueductoscomunitarios.co/ category/documentos/

Régimen de Servicios Públicos Domiciliarios (Ley 142 del $1^{\circ}$ de julio de 1994). (1994, julio 11). Diario Oficial, N. ${ }^{\circ} 41.433$.

Resolución 717. (2015, junio 22). Diario Oficial. 
Salamanca, J. (2001). El estado del arte de la regulación en el sector de agua potable y saneamiento básico en Colombia. Comisión de Regulación de Agua Potable y Saneamiento Básico (CRA).

Salas, M. E. (2007). Debate sobre la utilidad de la Metodología Jurídica: una reconstrucción crítica de las actuales corrientes metodológicas en la teoría del derecho. Isonomía, 27, 111-142.

Salazar Restrepo, B. (2019). La gestión comunitaria del agua en la municipalidad: un análisis comparado de experiencias en Envigado y Santuario, Antioquia [tesis de grado, Universidad de Antioquia]. http://tesis.udea.edu.co/ bitstream/10495/11160/1/SalazarBibiana_2019_GestionComunitariaAgua. pdf

Sandoval, D. (2014). Apuntes para una metodología de la historia crítica del derecho. Revista Brasileira de Estudos Políticos, 109, 139-176. https://doi. org/10.9732/P.0034-7191.2014v109p139

Sandoval-Moreno, A. y Günther, M. G. (2013). La gestión comunitaria del agua en México y Ecuador: Otros acercamientos a la sustentabilidad. Ra Ximhai, 9(2), 165-179. https://doi.org/10.35197/rx.09.02.e.2013.12.as

Sen, A. (1998). Capital humano y capacidad humana. Cuadernos de economía, 17(29), 67-72.

Tascón Varela, M. (2018). Exigibilidad del Derecho Humano del agua, en el Municipio de Restrepo, Valle del Cauca. Pontificia Universidad Javeriana de Cali.

Tobón, D. y Valencia, G. D. (2006). La participación privada en la provisión del servicio público de agua potable. El caso de cinco municipios de Antioquia. Universidad de Antioquia, Centro de Investigaciones Económicas.

Usquhart, S. y Mesquita, D. (2014). El agua como un derecho fundamental y el derecho al agua potable como un derecho humano fundamental, una propuesta teórica de políticas públicas. Revista Jurídicas, 11(1).

Zurbriggen, C. (2011). Gobernanza: Una mirada desde América Latina. Perfiles latinoamericanos, 19(38), 39-64.

Zurbriggen, C. (2014). Políticas latinoamericanas en la gestión del agua: de la gobernanza neoliberal a una gobernanza pública. Agua y territorio, 3, 89-99. https://doi.org/10.17561/at.vli3.1427 